\title{
LOCAL DEPLANATION OF DOUBLE REINFORCED BEAM CROSS SECTION UNDER BENDING*
}

\author{
Anguel Baltov, Ana Yanakieva \\ Institute of Mechanics, Bulgarian Academy of Science, \\ Acad. G. Bonchev St., Bl. 4, 1113 Sofia, Bulgaria, \\ e-mails: anguelbaltov@gmail.com, aniyanakieva@imbm.bas.bg
}

[Received 31 July 2015. Accepted 16 November 2015]

\begin{abstract}
Bending of beams, double reinforced by means of thin composite layers, is considered in the study. Approximate numerical solution is proposed, considering transitional boundary areas, where smooth quadratic transition of the elasticity modulus and deformations take place. Deplanation of the cross section is also accounted for in the areas. Their thickness is found equalizing the total stiffness of the cross section and the layer stiffness. Deplanation of the cross section of the transitional area is determined via the longitudinal deformation in the reinforcing layer, accounting for the equilibrium between the internal and the external moment, generated by the longitudinal stresses in the cross section. A numerical example is given as an illustration demonstrating model's plausibility. The model allows the design and the calculation of recycled concrete beams double reinforced by means of thin layers. The approach is in agreement with modern design of nearly zero energy buildings (NZEB). KEY WORDS: Concrete beams, reinforcing layers, approximate calculation model, NZEB.
\end{abstract}

\section{Introduction}

Combined systems of beams, reinforced by means of thin composite layers, are often used nowadays [1-5]. Usually, the reinforcing materials are composite polymers. They can also be special nano-materials, which are very effective, but too expensive. The reinforcing material is deposited as a very thin layer on the structural element. It has high strength, large modulus of elasticity and restricts the boundary deformation. Thus, deplanation of the

\footnotetext{
${ }^{*}$ Corresponding author e-mail: aniyanakieva@imbm.bas.bg

The study has been financially supported by the National Science Fund, Project DFNI E02/10 121214 .
} 
cross section takes place in the emerging boundary areas, which are also due to the technology of the boundary layer deposition. If the layer is plastered or pulverized, the reinforcing liquid diffusively penetrates the substrate material prior to hardening, creating a boundary area of high strength. If a reinforcing folio is stuck to the element surface, a restricting effect occurs and a transitional boundary area emerges by analogy to hydromechanics [6]. One can determine the thickness of that area, performing bending of simple beams, following the loading schemes in Fig. 3 and finding the maximal beam deflections. This has been performed in our previous paper [7].

We propose here a model of cross section deplanation in the boundary areas, Fig. 4. The boundary deformation is found from the equilibrium condition, equalizing the internal and the external moments. Consider the operation of double reinforced beams under bending. Then, deplanation clarification becomes an important practical issue. The material of the beam core can be concrete, steel fibre reinforced concrete, steel etc. We shall focus on a core, prepared from recycled concrete, conforming to modern tendencies of erecting nearly zero energy buildings and structures. Energy can also be saved by reducing the beam weight, as well as that of the structure supporting the beam. Thus, material together with resources needed for its preparation, can also be saved. An important issue is also the choice and employment of a deposition technology with less energy consumption.

\section{Elasticity modulus distribution in the transitional boundary} areas

Consider a bended beam operating in a linear elastic stage, where no macro cracks and separations occur. We assume there a smooth transition with a parabolic distribution of the elasticity modulus due to the small thickness of the transitional areas - see also [7], Fig. 1.

Consider specifically bending of a beam with a rectangular cross section, Fig. 2, with width band height $h$, thickness of the transitional boundary layer $\delta_{R}$ and thickness of the concrete core $2 y_{L}, y_{L}=\frac{h}{2}-\delta_{R}$. The distribution of the elasticity modulus in the transitional layer follows a parabolic law.

(1) $\quad E(\bar{y})=E_{C}+\frac{\Delta E_{R}}{\delta_{R}^{2}} \bar{y}^{2}, \quad \Delta E_{R}=E_{R}-E_{C}, \quad \bar{y}=y-y_{L}, \quad \bar{y} \in\left[0, \delta_{R}\right]$.

Here, $E_{C}$ is the elasticity modus of the concrete core and $E_{R}$ is that of the thin reinforcing layer, Fig. 1. Equation (1) satisfies the conditions of smooth transition: 


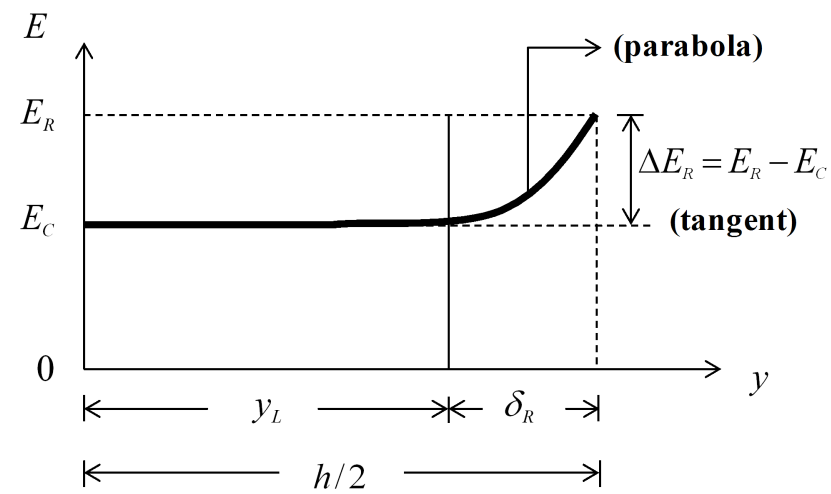

Fig. 1. Elasticity modulus $E$ distribution in the cross section

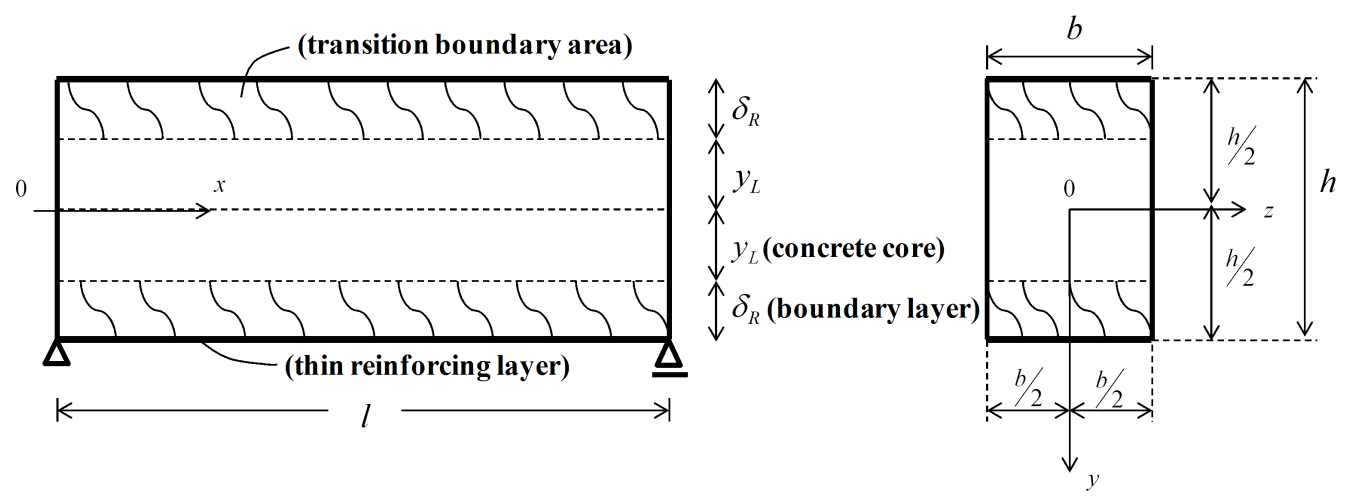

Fig. 2. A beam with rectangular cross section

for $y \in\left[0, y_{L}\right], E=E_{C}$,

for $y=y_{L}, E=E_{C}, \frac{d E}{d y}=0$,

for $y=\frac{h}{2}, E=E_{R}$.

To find $\delta_{R}$ in the case of a diffusing reinforcing liquid is an extremely difficult task, due to the nonlinearity of the diffusion coefficient and the nonlinear dependence of the elasticity modulus on the liquid concentration. Those quantities can be found experimentally, only. There is no such problem in other technologies (folio sticking etc.). Hence, we proved in [7] the possibility to find $\delta_{R}$ directly from the stiffness of the thin reinforced beam, using one of the experimental set-ups in Fig. 3. 


\section{Stiffness determination.}

We assume that beam maximal deflection $f$ can be experimentally found, following one of the schemes shown in Fig. 3.
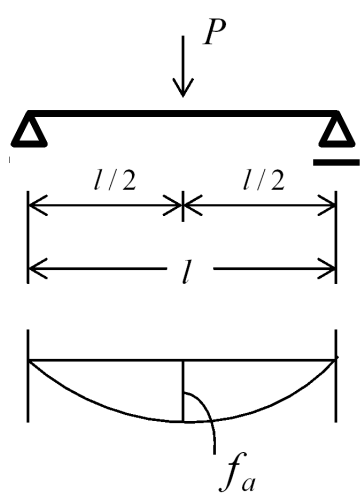

a)

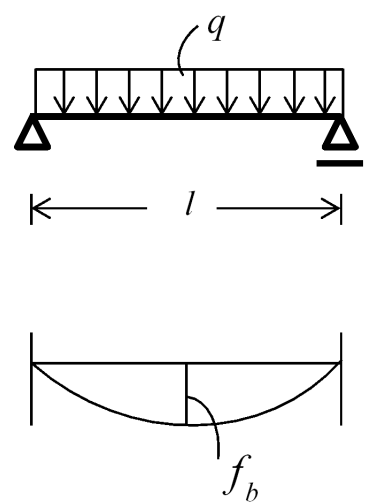

b)
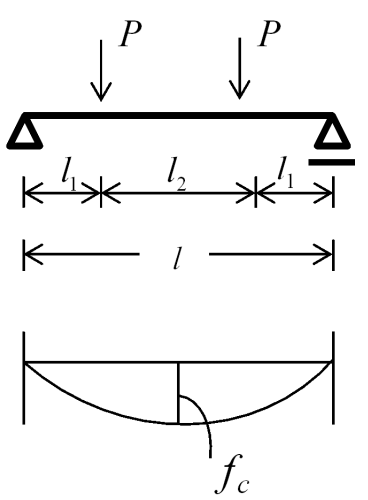

c)

Fig. 3. Experimental schemes of simple beams subjected to three different types of loading

Due to beam static determinacy, the maximal deflection $f$ is experimentally found via the known formulas of the structural mechanics, but involving stiffness $K_{f}$ of the compound beam. For instance, $f_{b}=\frac{q l^{4}}{48 K_{f}}$ i.e. $K_{f}=\frac{q l^{4}}{48 f_{b}}$ in scheme (b).

By definition, the stiffness of a compound beam is:

$$
\begin{aligned}
& \frac{1}{2} K_{f}=K_{R}+K_{L}, \quad K_{f}=2 b \int_{0}^{h / 2} E(y) y^{2} d y, \\
& K_{L}=\frac{b y_{L}^{3}}{3} E_{C}, \quad K_{R}=\sum_{i=1}^{3} \chi_{i}^{\prime} \delta_{R}^{i},
\end{aligned}
$$

for

$$
\begin{aligned}
\chi_{1}^{\prime} & =\frac{b h^{2}}{12}\left(2 E_{C}+E_{R}\right), \\
\chi_{2}^{\prime} & =-\frac{b h}{12}\left(5 E_{C}+E_{R}\right), \\
\chi_{3}^{\prime} & =\frac{b}{10}\left(3 E_{C}+\frac{1}{3} E_{R}\right) .
\end{aligned}
$$


The experimentally found stiffness $K_{f}$, using the maximal deflection $f$ of the compound beam is constant, and it can be used in the analysis of bending of any beam thus reinforced.

\section{Thickness of the transitional boundary layer determination}

Based on $K_{f}$ found, considering the known deflection $f$, we can equalize both stiffnesses obtaining a complete 3 -d degree algebraic equation to determine the thickness of the transitional boundary layer $\delta_{R}$ :

$$
\sum_{i=0}^{3} \chi_{i}^{\prime \prime} \delta_{R}^{i}=0
$$

where:

$$
\begin{aligned}
\chi_{0}^{\prime \prime} & =-\frac{1}{2} K_{f}+\frac{b h^{3}}{24} E_{C}, \\
\chi_{1}^{\prime \prime} & =\chi_{1}^{\prime}-\frac{b h^{2}}{4} E_{C}, \\
\chi_{2}^{\prime \prime} & =\chi_{2}^{\prime}+\frac{b \hbar}{2} E_{C}, \\
\chi_{3}^{\prime \prime} & =\chi_{3}^{\prime}-\frac{b}{3} E_{C} .
\end{aligned}
$$

The result for $\delta_{R}$ enables one to determine the cross section deplanation using the condition of moment equilibrium.

\section{Cross section deplanation of the bended beam transitional boundary area}

Cross section deplanation takes place due to beam reinforcement and emergence of a transitional boundary layer, Fig. 4.

Assume that beam core bends in conformity with the hypothesis of planar cross sections and deplanation takes place within the transitional boundary layer, only.

For $y \in\left[0, y_{L}\right]: \varepsilon(x, y)=\psi(x) y, \psi(x)=w^{\prime \prime}(x)$ is stiffness of the bended axis and $w(x)$ is beam deflection along the longitudinal axis $O(x)$.

For $y \in\left[y_{L}, \frac{h}{2}\right]$, assume approximately that deplanation takes place evenly, with a smooth transition for $y=y_{L}$ and being parabolic, as a first approximation. The small thickness of the transitional boundary layer $\delta_{R}$ verifies such an assumption.

$$
\varepsilon(x, y)=a_{0}+a_{1} y-a_{2} y^{2} .
$$




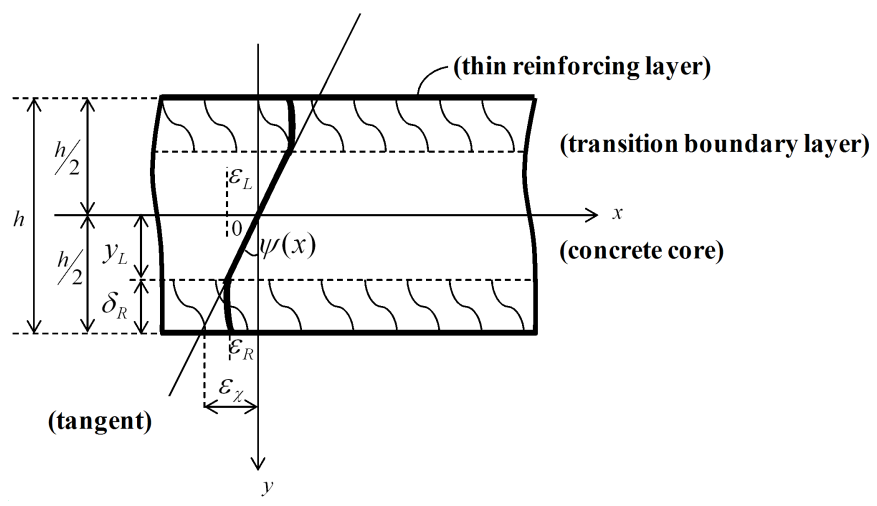

Fig. 4. Cross section deplanation in the transitional area

The sign before $a_{2}$ is negative due to the sign of the cross section curvature. Coefficients $a_{i},(i=0,1,2)$ depend on $x$, only. Smooth transition is realized by the conditions:

For $y=y_{L}, \varepsilon=\varepsilon_{L}=\psi(x) y_{L}$ and $\left.\frac{d \varepsilon}{d y}\right|_{y=y_{L}}=\psi(x)$.

For $y=\frac{h}{2}, \varepsilon=\varepsilon_{R}$, which will be found from the equilibrium condition.

Expression (5) can be presented in the local coordinate system $(x, \bar{y})$, $\bar{y}=y-y_{L}, y_{L}=\frac{h}{2}-\delta_{R}$, for convenience, where $\delta_{R}$ is the already found thickness of the transitional boundary layer. For $y \in\left[y_{L}, \frac{h}{2}\right], \bar{y} \in\left[0, \delta_{R}\right]$ the expression reads:

$$
\varepsilon(x, \bar{y})=\sum_{i=0}^{2} \bar{a}_{i} \bar{y}^{i},
$$

where:

$$
\bar{a}_{0}=\varepsilon_{L}=\psi(x) y_{L}, \quad \bar{a}_{1}=\psi(x), \quad \bar{a}_{2}=\frac{\psi(x)}{\delta_{R}}-\frac{\Delta \varepsilon_{R}}{\delta_{R}^{2}}, \quad \Delta \varepsilon_{R}=\varepsilon_{R}-\varepsilon_{L} .
$$

\section{Stress distribution within the transitional boundary area}

Note the initial assumption that the beam operates in the linear-elastic stage to find distribution of stresses normal to the cross section of the transitional boundary area. Then, stresses are defined considering strains and the elasticity moduli in conformity with Hooke's law: 
For $y \in\left[0, y_{L}\right], E(y)=E_{C}$ and hence:

$$
\sigma(x, y)=E_{C} \psi(x) y,
$$

For $y \in\left[y_{L}, \frac{h}{2}\right]$ or $\bar{y} \in\left[0, \delta_{R}\right]$, rewriting the expression for stresses, we obtain:

$$
\sigma(x, \bar{y})=E(\bar{y}) \varepsilon(x, \bar{y})=\sum_{i=0}^{4} C_{i}(x) \bar{y}^{i}
$$

where:

$$
\begin{aligned}
C_{0}(x) & =E_{C} \varepsilon_{L}\left(x, y_{L}\right)=E_{C} \psi(x) y_{L}, \\
C_{1}(x) & =E_{C} \psi(x), \\
C_{2}(x) & =\left(\frac{1}{\delta_{R}^{2}} \varepsilon_{L}\left(x, y_{L}\right) \Delta E_{R}-\frac{1}{\delta_{R}} \psi(x) E_{C}\right)+\Delta \varepsilon_{R}\left(\frac{E_{C}}{\delta_{R}^{2}}\right)=C_{2}^{\prime}+\Delta \varepsilon_{R} C_{2}^{\prime \prime}, \\
C_{3}(x) & =\psi(x) \frac{\Delta E_{R}}{\delta_{R}^{2}}, \quad \Delta \varepsilon=\varepsilon_{R}-\varepsilon_{L}, \\
C_{4}(x) & =\frac{\Delta \varepsilon_{R}}{\delta_{R}^{4}} \Delta E-\frac{\Delta E_{R}}{\delta_{R}^{3}} \psi(x) .
\end{aligned}
$$

The smoothness of the transition of $\varepsilon(x, y)$ and $E(y)$ helps to the smoothness of the transition of $\sigma(x, y)$ within the boundary layer, i.e.:

For $y=y_{L}, \sigma=\sigma_{L}=E_{C} \varepsilon_{L}=E_{C} \psi(x) y_{L}$.

For $y=\frac{h}{2}, \sigma=\sigma_{R}=E_{R} \varepsilon_{R}$.

Strain $\varepsilon_{R}$ along the edge is unknown but it can be found from the equilibrium between the external and internal moments. Using the model, one should check whether $\sigma_{L} \leq \sigma_{c r}^{C}$, where $\sigma_{c r}^{C}$ is the allowable stress of concrete tension related to concrete tension strength. One should also check whether $\sigma_{R} \leq \sigma_{c r}^{R}$, where $\sigma_{c r}^{R}$ is the allowable stress of the thin reinforcing layer.

\section{Edge strain determination in the thin reinforcing layer}

However, the edge strain $\varepsilon_{R}$ in the reinforcing thin layer was left unfound. To find it, we use the condition of equilibrium between the external beam moments $M(x)$ and the internal ones, generated by the normal stresses. We consider a simplified case disregarding the axial force:

$$
M(x)=2 b \int_{0}^{h / 2} \sigma(x, y) y d y=M_{L}(x)+M_{R}(x),
$$


where:

$$
\begin{aligned}
& M_{L}(x)=2 b \int_{0}^{y_{L}} \sigma(x, y) y d y=2 b E_{C} \psi(x) \frac{1}{3} y_{L}^{3}, \\
& M_{R}(x)=2 b \int_{y_{L}}^{h / 2} \sigma(x, y) y d y=2 b \int_{0}^{\delta_{R}} \sigma(x, \bar{y})\left(\bar{y}+y_{L}\right) d \bar{y} .
\end{aligned}
$$

The transformation of the above relations yields:

$$
\frac{M_{R}(x)}{2 b}=L(x)-\Delta \varepsilon_{R} Q(x), \quad M_{R}(x)=M(x)-M_{L}(x),
$$

for $L(x)=\sum_{i=0}^{4} D_{i}(x)\left[\frac{\delta_{R}^{i+2}}{(i+2)}+y_{L} \frac{\delta_{R}^{i+1}}{(i+1)}\right], Q(x)=\Delta E_{R}\left(\frac{1}{4}+\frac{y_{L}}{3 \delta_{R}}\right)$, where:

$$
\begin{aligned}
& D_{0}=E_{C} \psi(x) y_{L}, \quad D_{1}=E_{C} \psi(x), \\
& D_{2}=E_{C} \psi(x) \frac{1}{\delta_{R}}+\frac{\Delta E_{R}}{\delta_{R}^{2}} \psi(x) y_{L}, \\
& D_{3}=\frac{\Delta E_{R}}{\delta_{R}^{2}} \psi(x), \quad D_{4}=\frac{\Delta E_{R}}{\delta_{R}^{3}} \psi(x) .
\end{aligned}
$$

Considering equations (8), (9) and (10), we obtain:

$$
\varepsilon_{R}=\varepsilon_{L}+\frac{\bar{L}(x)}{Q(x)} \text { for } \bar{L}(x)=L(x)+E_{C} \psi(x) \frac{y_{L}^{3}}{3}-\frac{M(x)}{2 b} .
$$

The degree of approximation of the proposed model can be verified by an experimental determination of the edge strains $\varepsilon_{R}$, using tension transducers and comparison of the results to the calculated ones according to expression (12).

\section{Numerical example. Parametric analysis}

Consider the experiments of [8]. We look for an equivalent double reinforced concrete beam, which should have ratio $\frac{q}{f}$ identical to that of the concrete steel fibre reinforced beam in [8], following loading scheme (b) in Fig. 3. Data are as follows: $b=0.305 \mathrm{~m}$ and $h=0.768 \mathrm{~m}, l=5.69 \mathrm{~m} ; E_{C}=1.7 \times 10^{10}$ $\mathrm{N} / \mathrm{m}^{2}, E_{R}=13.1 \times 10^{10} \mathrm{~N} / \mathrm{m}^{2}$. We showed in [7] that a 3 -d degree equation for $\delta_{R}$ is derived on that basis:

$$
-\chi+4,9 \delta_{R}-6,4 \delta_{R}^{2}+3,3 \delta_{R}^{3}=0,
$$


where:

$$
\chi=100\left(\frac{K_{f}}{2 b \Delta E_{R}}-\frac{E_{C} h^{3}}{24 \Delta E_{R}}-\right), \quad K_{f}=\frac{q l^{4}}{48 f} .
$$

Equation (14) shows that the relation between parameter $\chi$ and $\delta_{R}$ is a 3 -d degree polynomial. We perform a parametric analysis and find the relation between the load-deformation parameter $\frac{q}{f}$ and the thickness of the transitional boundary layer $\delta_{R}$, Table 1 .

Table 1. Relation between the load-deformation parameter and the thickness

\begin{tabular}{|c|c|c|}
\hline$\frac{q}{f} \cdot 10^{8}\left[\mathrm{~N} / \mathrm{m}^{2}\right]$ & $\chi\left[\mathrm{m}^{3}\right]$ & $\delta_{R}[\mathrm{~m}]$ \\
\hline 0.10 & 0.200 & 0.043 \\
\hline 0.20 & 0.345 & 0.078 \\
\hline 0.25 & 0.400 & 0.081 \\
\hline
\end{tabular}

Data in Table 1 are plotted in Fig. 5.

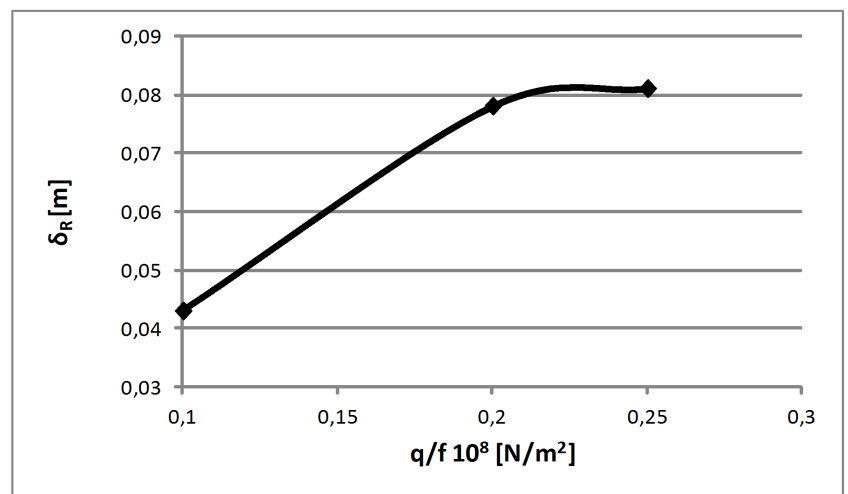

Fig. 5. Relation between $\frac{q}{f}$ and $\delta_{R}$

Figure 5 shows that the thickness of the transitional layer $\delta_{R}$ increases with the increase of the load-deformation parameter $\frac{q}{f}$. The example proves that the assumed model produces plausible results.

\section{Conclusion}

The presented calculation model of bending of a double reinforced concrete beam yields a closed form solution, which enables the designer to perform a fast and efficient analysis. 
The assumed parabolic approximations can be further complicated, if necessary.

The model is based on easily realized experiments, where the maximal deflection can be measured accurately enough.

The use of the calculation model in the design of double reinforced beams fabricated from recycled concrete may facilitate the erection of nearly zero energy buildings.

\section{REFERENCES}

[1] Lorenzis, L., B. Miller, A. NAnni. Bond of FRP Laminates to Concrete. ACI Materials Journal, 98 (2001), No. 3, 256-264.

[2] Shen, N., J. Teng, J. Yang. Interfacial Stresses in Beams and Slabs Bonded with Thin Plate.ASCE Journal of Engineering Mechanics, 127 (2001), No. 4, 399-406.

[3] Rabinovich, O., Y. Frostig. Delamination Failure of RC Beams Strengthening with FRP Strips - a Closed-form High-order and Fracture Mechanics Approach. ASCE Journal of Engineering Mechanics, 127 (2001), No. 8, 852-862.

[4] Nikolova, G. The Effect of Temperature, Material Properties of Adhesive Layer and Geometry on a Bi-material Structure. Comptes rendus de l'Academie bulgare des Sciences, 68 (2015), No. 3, 375-382.

[5] Nikolova, G., J. Ivanova, V. Valeva, Z. Mroz. Mechanical and Thermal Loading of Two-plate Structure. Comptes rendus de l'Academie bulgare des Sciences, 60 (2007), No. 7, 735-742.

[6] Sinaiski, E. G., M. Braun (Translator). Hydromechanics: Theory and Fundamentals, Ed. Wiley-VCH, 2011.

[7] Baltov, A., A. Yanakieva. Bending of Concrete Beams Reinforced via Thin Layers, In Proc.: XV Inter. Scientific Conf. VSU, Bulgaria, Sofia, 2015, I-73-78.

[8] Dinev, D. Investigation on Constructions working on Bending, Reinforced by Composite Materials, PhD Thesis for Degree Doctor, Sofia, 2006. 